\title{
Identification of Severe Multiple Contingencies in Electric Power Networks
}

\author{
Vaibhav Donde ${ }^{\dagger}$, Member, IEEE, Vanessa López ${ }^{\ddagger}$, Bernard Lesieutre ${ }^{\dagger}$, Member, IEEE, Ali Pinar ${ }^{\ddagger}$, \\ Member, IEEE, Chao Yang ${ }^{\ddagger}$, and Juan Meza
}

\begin{abstract}
In this paper we propose a two-stage screening and analysis process for identifying multiple contingencies that may result in very severe disturbances and blackouts. In a screening stage we form an optimization problem to find the minimum change in the network to move the power flow feasibility boundary to the present operating point and that will cause the system to separate with a user-specified power imbalance. The lines identified by the optimization program are used in a subsequent analysis stage to find combinations that may lead to a blackout. This approach is applied to a 30-bus system with encouraging results.
\end{abstract}

Index Terms-Power System Security, Graph Partitioning

\section{INTRODUCTION}

In this paper we propose a two-stage screening and analysis process for identifying multiple contingencies that may result in very severe disturbances and blackouts. Our work is motivated by the occurrences of such events and the complete lack of practical algorithms to anticipate them. As a recent example, the August 14, 2003 blackout in the northeast of the U.S. resulted in a loss of estimated $61.8 \mathrm{GW}$ of electric load and affected 50 million people [1]. The cost associated with this blackout was about $\$ 6$ billion as estimated by the U.S. Department of Energy (DOE) [2]. While many factors contributed to the prevailing operating conditions on that afternoon, just three transmission lines that underwent faults and subsequent outages in relatively short succession initiated the blackout process. These line outages irreversibly overloaded the system and resulted in a very fast and dramatic blackout.

Identification of such lines, removals of which eventually leads to a failure, is crucial to power system security. The notion of " $n-k$ " security is traditionally employed to identify the weakest components in a power system network. Conceptually, focusing only on lines for the moment, in a system having $n$ transmission lines, $k$ lines are removed from service to test whether a power flow solution exists for the

This work was supported by the Director, Office of Science, of the U.S. Department of Energy under Contract No. DE-AC02-05CH11231.

†Vaibhav Donde (VDonde@lbl.gov) and Bernard Lesieutre (BCLesieutre@lbl.gov) are with the Environmental Engineering Technologies Division, Lawrence Berkeley National Laboratory, Berkeley, CA 94720.

*Vanessa López (VLLopez@lbl.gov), Ali Pinar (APinar@lbl.gov), Chao Yang (CYang@lbl.gov) and Juan Meza (JCMeza@lbl.gov) are with the Computational Research Division, Lawrence Berkeley National Laboratory, Berkeley, CA 94720. new network topology. A process of analysis based on complete enumeration becomes computationally prohibitive for large systems as $k$ takes values greater than 2 .

More sophisticated analysis tools are thus required that can efficiently handle the issue of computational burden that the abovementioned method of enumeration encounters. This paper proposes one such approach that exploits results from spectral graph theory and it is cast as an optimization problem. Using a two-stage approach we identify a few transmission lines in a large power system network (graph) which, when cut, cause the power flow equations to not have a solution, possibly leading to a failure. With the initial screening stage approach we use, the graph is partitioned into subgraphs and the lines in the cut-set may be seen as elements of the network posing a threat to power system security. A subsequent detailed analysis stage is then performed to identify those lines in the cut-set (stage 1) whose removal will result in a loss of feasibility.

Our approach is partially motivated by the work of Alvarado, Dobson and $\mathrm{Hu}$ [3]. In their work they computed the closest point on the feasibility boundary to the present operating point. That is, the minimum change in power injections that would result in operation at the edge of feasibility. In our approach we ask: what is the smallest change in line characteristics that will move edge of the feasibility region to the present operating point. Note that for a given network topology, power flows have a continuous dependence on bus voltages $V$ and phase angles $\theta$. When transmission lines are removed from the network, the power flows change abruptly due to the changed network topology. Line outages thus introduce a discrete flavor into power flow equations. We employ a relaxation that allows partial line outages. Examining partial line outages we can identify conditions when the feasibility region contracts to the present operating point. This does not immediately provide the final solution to our problem of vulnerability identification, although it does provide a screening process to identify potentially important lines for further finer analysis. Lines identified during the screening process are typically few in number, which are then amenable to enumeration tool for " $n$ $k$ ” security analysis or more sophisticated techniques such as combinatorial algorithms.

The paper is organized as follows. Section II summarizes graph theory preliminaries that provide a theoretical foundation for the mathematical formulation of the problem 
described in Section III. Application to the IEEE 30 bus system is presented in Section IV. Concluding remarks are included in Section V.

\section{SPECTRAL GRAPH THEORY PRELIMINARIES}

A review of concepts from spectral graph theory that are relevant to our work follows. For a thorough treatment of the subject see, for example, $[4,5]$. Given a graph $\mathrm{G}$ with $m$ nodes and $n$ directed edges or branches, the branch-node incidence matrix $A$ of $\mathrm{G}$ is an $n$-by- $m$ matrix with

$$
\begin{aligned}
& A_{i, j}=1 \text { if branch } i \text { originates at node } j, \\
& A_{i, j}=-1 \text { if branch } i \text { ends at node } j, \\
& A_{i, j}=0 \text { otherwise. }
\end{aligned}
$$

Note that the sum of the elements in each row of $A$ is equal to zero, so the vector $e=\left[\begin{array}{llll}1 & 1 & \ldots & 1\end{array}\right]^{\mathrm{T}}$ is in the null space of $A$ (i.e., $A e=0)$.

The Laplacian of the graph $\mathrm{G}$ is defined as the matrix $A^{\mathrm{T}} A$. If nonnegative weights $\beta_{\mathrm{i}}, i=1, \ldots, n$, are associated with the edges of the graph $\mathrm{G}$, the weighted Laplacian is similarly defined as

$$
L_{G}=A^{T} \operatorname{diag}(\beta) A,
$$

where $\beta$ is a vector with its $\mathrm{i}$-th element equal to $\beta_{\mathrm{i}}$. Note that a zero weight corresponds to two disconnected nodes in the graph. Furthermore, the Laplacian $A^{\mathrm{T}} A$ is a special case of the weighted Laplacian, hence in what follows we only refer to a weighted Laplacian.

A weighted Laplacian matrix $L_{\mathrm{G}}$ has real, nonnegative eigenvalues. Note that zero is always an eigenvalue with corresponding eigenvector $e=\left[\begin{array}{llll}1 & 1 & \ldots & 1\end{array}\right]^{\mathrm{T}}$, since $A e=0$. Now, if the graph $\mathrm{G}$ is disconnected, then the spectrum of $L_{\mathrm{G}}$ is given by the union of the spectra of the Laplacian matrices of the subgraphs, or connected components, of G. In particular, since the weighted Laplacian of each subgraph has a zero eigenvalue, it follows that the multiplicity of the zero eigenvalue of $L_{\mathrm{G}}$ is equal to the number of connected components of G. Furthermore, information about the decomposition of the nodes of $G$ into its connected components may be extracted from the eigenvectors corresponding to the zero eigenvalue of $L_{\mathrm{G}}$, as described next.

Let us denote the number of connected components of $\mathrm{G}$ by $\eta$ and define a set of vectors $v_{i}, i=1, \ldots, \eta$, such that the $\mathrm{j}$-th element of $v_{i}$ is equal to 1 if node $\mathrm{j}$ is in the $\mathrm{i}$-th connected component, and 0 otherwise. Note that the vectors $v_{i}$ form an orthogonal basis of eigenvectors corresponding to the zero eigenvalue of $L_{\mathrm{G}}$. A linear combination of the eigenvectors $v_{i}$, viz., $\sum_{i=1, \ldots, \eta} \sigma_{i} v_{i}$, provides $e$ as an eigenvector, as expected, by setting each coefficient $\sigma_{i}$ to 1 . On the other hand, by setting $\sigma_{k} \neq \sigma_{j}$ for $k \neq j$, the linear combination results in an eigenvector that separates out the nodes of $\mathrm{G}$ into its connected components. Note that such eigenvector has identical elements at entries corresponding to nodes within a connected component. We denote this eigenvector as $w$ for purpose of the discussion in Section III.

\section{PRoblem Formulation}

This section discusses the power system model and mathematics underlying the process for identifying lines in a power network to which power system security is sensitive. We propose a two-stage process in which an initial screening stage (the main focus of this paper) is used to find a small set of candidate lines to be considered in a subsequent analysis stage, to identify combinations of line outages that may result in a severe disturbance. In the screening stage several modeling simplifications are used to allow tractable analysis, including neglecting losses and ignoring issues related to reactive power and voltage. It is possible to use a detailed model in the analysis (or second) stage and it may be beneficial to include dynamic analysis. In this, our initial report on our work, we focus on a static power flow analysis, and intend to extend our results to more detailed models in our continuing work.

\section{A. Power System Model and Relaxation}

Consider a lossless power system network having $m$ buses (nodes) and $n$ lines (edges). Voltages at the buses are assumed to be fixed at their nominal values, i.e., 1 p.u. This assumption may be justified by the fact that the screening stage of analysis is only expected to identify a small number of lines to be considered for a detailed analysis at the later stage. Thus a simplistic view of a power system is sufficient for filtering out lines which do not play an important role in breaching system security.

As bus voltages are fixed to 1 p.u., dependence of real power injections at buses on the network angle variables $\theta$ is fully described by active power flow constraints. Reactive power flow constraint is then unnecessary for our purpose. We represent real power flow equations in matrix form as it immediately brings in the aspects of the graph theory we need for analysis. Letting $B$ denote a diagonal matrix with the values of line admittances ${ }^{1}$ on its diagonal, the power flowing through the lines can be compactly expressed as

$$
P_{\text {line }}=B \sin (A \theta) \text {, }
$$

where $P_{\text {line }}$ is a vector of power flows over the lines and $A$ is a branch-node incidence matrix of the graph. A vector of power injections $P$ is then obtained by adding up the powers flowing out of the buses into the network. This is represented in matrix form as

$$
A^{T} B \sin (A \theta)-P=0
$$

with $A \theta$ taking values between $-\pi / 2$ and $+\pi / 2$ radians, as required for steady state stability.

Now consider a transmission line connecting any two buses. Reducing its admittance to zero is equivalent to removing that line from service. Thus admittances effectively provide a way to represent line outages by associating with

\footnotetext{
${ }^{1}$ We assume a lossless system and consider lines that appear inductive, not capacitive.
} 
them a set of variables $\gamma_{i}, i=1, \ldots, n$, that can be used to indicate whether or not the $\mathrm{i}$-th line is in service. For instance, the value of a modified admittance can be defined as the product of the admittance of line $i$ with $\left(1-\gamma_{i}\right)$. The modified admittance is then equal to the nominal admittance when $\gamma_{i}=0$, i.e., when the line is in service. On the other hand, it is zero when $\gamma_{i}=1$, which corresponds to the i-th line being removed from service. The power flow model (1), now with modified admittances, can thus be represented as

$$
A^{T} B(I-\Gamma) \sin (A \theta)-P=0,
$$

where $\Gamma$ is a diagonal matrix having $\gamma_{i}$ on its i-th diagonal entry and $I$ is the identity matrix.

It should be noted that $P$ is regarded as fixed and that the variables in $(2)$ are $\operatorname{diag}(\Gamma)$ and $\theta$. Elements on the diagonal of $\Gamma$ take on discrete values, either 1 or 0 , representing operating status of the corresponding line. Figure 1 shows the schematic view of (2) in $P$ space. When the line under consideration is in service, the curve shown by a solid line represents a feasibility boundary for the power flow constraint. The system operating point lies inside the feasible region. When the line is removed from service, the feasibility boundary comes closer to the operating point, making it more vulnerable to infeasibility or failure. It may so happen that removal of the line pushes the boundary past the operating point (dotted-lined curve), making system operation infeasible due to nonexistence of a power flow solution. Thus removing lines from the network effectively shifts the boundary of the feasibility region of solutions to (2), for a given operating point $P$.

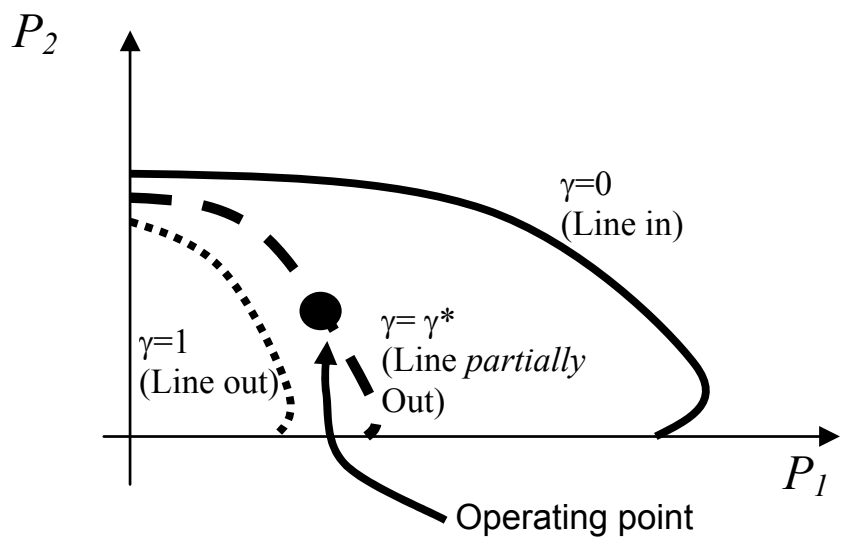

Figure 1: Space of real power injections showing feasibility boundaries for various line statuses.

Referring to Figure 1, it is apparent that had $\gamma$ been able to change continuously in the interval $[0,1]$, the feasibility boundary could pass exactly through the operating point and the system failure will just occur. This situation is shown by a dashed curve representing the feasibility boundary. The power flow solution just vanishes and this occurs when the line is partially taken out of service with $\gamma=\gamma^{*}$. In the screening stage of our analysis, we allow such partial line outages to occur. Equivalently, we relax the constraint that $\gamma_{i}$ is a discrete variable taking values of 1 or 0 , and allow it to take continuous values in the interval $[0,1]$. This makes the real power flow constraint equation (2) continuous in both $\theta$ and $\gamma$.

Now, the Jacobian $\mathrm{J}$ of (2) with respect to $\theta$,

$$
J=A^{T} B(I-\Gamma) \operatorname{diag}(\cos (A \theta)) A,
$$

is identical in structure to a weighted Laplacian (Section II) since the entries on the diagonal of the matrix $B(I-$ $\Gamma) \operatorname{diag}(\cos (A \theta))$ are nonnegative (recall that $|A \theta| \leq \pi / 2)$.

Thus, $J$ has a zero eigenvalue. This is also consistent with the fact that the power system network under consideration is (initially) connected.

It is worth noting that when the power flow equation for a reference (slack) bus is removed from (2), along with its bus variable $\theta$, the resulting (reduced order) Jacobian does not have a zero eigenvalue. Moreover, it does not have the structure of a weighted Laplacian. This reduced order Jacobian is singular only when the operating point lies on the feasibility boundary [3].

We preserve the network structure by not omitting the reference bus in order to be able to draw direct analogies with spectral graph theory and use the latter in our study. In our formulation, the Jacobian $J$, which is given by (3), is always singular with a single, trivial zero eigenvalue and corresponding eigenvector $e=\left[\begin{array}{llll}1 & 1 & \ldots & 1\end{array}\right]^{\mathrm{T}}$. Nevertheless, the zero eigenvalue of $J$ has multiplicity greater than one when the operating point lies on the feasibility boundary. We use this criterion to ensure the vanishing power flow solution. A zero eigenvalue of $J$ with multiplicity greater than one also means that the graph has been fragmented into subgraphs and, further, the buses in each subgraph can be discerned from the eigenvectors corresponding to the zero eigenvalue (Section II).

\section{B. A Constrained Optimization Problem}

Conceptually, the problem we pose using the relaxed model is as follows. We aim to identify a small set of lines in the network whose (possibly partial) removals from service will make the feasibility boundary pass through the operating point $P$. At a solution to this problem, the system is on the verge of infeasibility. Complete removals of the identified lines may result in an immediate and dramatic system failure. We also need a mechanism to ensure that the resulting blackout is severe in some sense. In Section III-C we define a measure for blackout severity that is suitable for our purposes.

Mathematically, the problem may be described in a constrained optimization framework as

subject to:

$$
\min _{\gamma, \theta, w} \operatorname{trace}(\Gamma)
$$

$$
\begin{aligned}
& A^{T} B(I-\Gamma) \sin (A \theta)-P=0 \\
& w^{T} J=0 \\
& w^{T} e=0 \\
& w^{T} w=1
\end{aligned}
$$




$$
\begin{aligned}
& 0 \leq \gamma_{i} \leq 1, i=1, \ldots, n \\
& -\pi / 2 \leq A \theta \leq \pi / 2,
\end{aligned}
$$

where $\Gamma=\operatorname{diag}(\gamma)$, as defined in (2), and the Jacobian $J$ is as in (3). Constraint (5) ensures that $J$ is singular and (6) guarantees that this singularity is due to a non-trivial zero eigenvalue by requiring $w \neq e$. Thus constraints (5)-(6), along with (4), place the operating point $P$ on the feasibility boundary. The use of the normalization (7) for the eigenvector will become apparent in Section III-C, where we introduce a measure for the severity of a disturbance. Finally, constraint (9) is required to ensure a steady state stable operation of the power network.

Recall from Section II that removal of a line appears in a weighted Laplacian as a zero weight. This is observed to happen from (3) whenever an element in the vector $\gamma$ equals 1 (direct removal of a line from the network), or when equality holds in (9), which corresponds to maximum possible power flowing through a line. The multiplicity of the zero eigenvalue of $J$ gives us the number of subgraphs the graph is partitioned into, and the buses in each subgraph can be determined from the eigenvector $w$, as explained in Section II.

An important issue now is to assess the severity of the disturbance resulting after the identified lines are taken out of service, in terms of the arguments of the optimization problem so that the optimization may be simultaneously constrained to identify solutions only corresponding to severe blackouts.

\section{Severity of System Failure}

Blackout severity may be measured in various ways [6]. As the formulation described in Section III-B seeks to partition the network into groups in order to cause a failure, shortage or surplus of power within the resulting groups is a natural way to define a measure of severity for our purpose. In the case when the network is partitioned into two groups, the eigenvector $w$ in (5)-(7) can be used to define such a measure of severity. In a two-group situation, a power imbalance measure of severity of the disturbance is then related to the shortage of power in one group after partitioning, which is identical in magnitude with the excess of power in the other group after partitioning, and with the net power flowing over the partition cut-set before partitioning. The derivation of the expression for disturbance severity in a two-group case follows.

Referring to Figure 2, suppose there are $m$ buses in the network and that the corresponding graph is partitioned into two subgraphs, one with $k$ nodes and the other with $m-k$ nodes. Let $S$ denote the sum of power injections corresponding to the buses in group 1. Then, by the conservation of power, the sum of the power injections in group 2 is $-S$. Since the elements in the eigenvector $w$ corresponding to nodes within a group are identical, assume, without loss of generality, that $w$ has $k$ elements equal to $(m-$ $k) / c$ and $m-k$ elements equal to $-k / c$, where $c$ is a constant to be determined. Note that constraint (6) is satisfied for such $w$ and that from (7) it follows that $c=\sqrt{k m(m-k)}$.

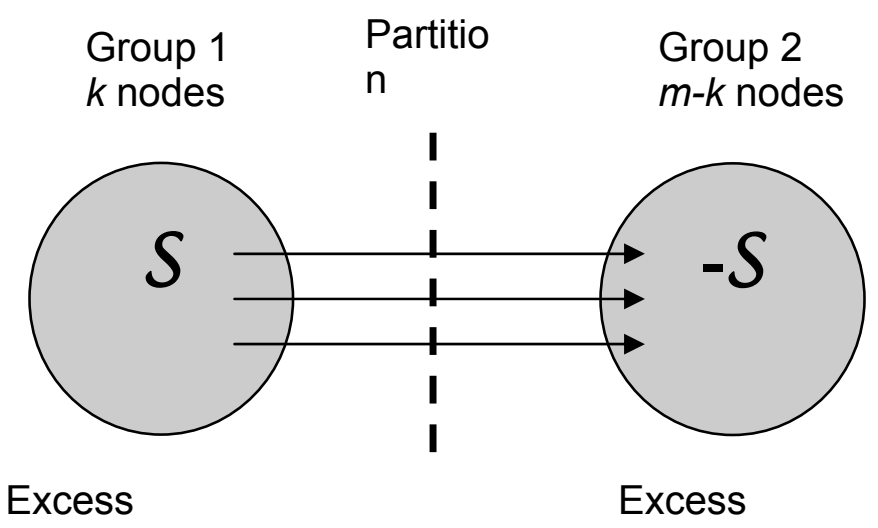

Figure 2: Power system network partitioned into two groups, one with excess generation and the other with excess load.

The aim here is to obtain $S$ in terms of $w$ and $P$. Consider the inner product of vectors $w$ and $P$,

$\left(w^{\mathrm{T}} P\right)^{2}=\{((m-k) / c)($ total power injection in group 1) + $(-k / c)$ (total power injection in group 2) $\}^{2}$

$$
\begin{aligned}
& =\{((m-k) / c) S+(-k / c)(-S)\}^{2} \\
& =m^{2} S^{2} / c^{2} .
\end{aligned}
$$

Also,

$$
\sum_{i}\left(w_{i}\right)^{4}=m^{2} / c^{2}-3 / m .
$$

Solving for $S^{2}$ in (10) and $m^{2} / c^{2}$ in (11), one can express $S^{2}$ in terms of $w$ and $P$ as

$$
\operatorname{severity}(w, P) \equiv S^{2}=\frac{\left(w^{T} P\right)^{2}}{\sum_{i}\left(w_{i}\right)^{4}+3 / m} .
$$

Therefore, when a solution to the constrained optimization problem partitions the graph into two subgraphs, the eigenvector $w$ gives a perfect measure of the severity of the power imbalance resulting from the removal of lines identified by the solution. The definition of severity as in (12) does not require knowledge of nodes within the subgraphs a priori, hence we append the additional constraint

$$
s_{\text {min }} \leq \operatorname{severity}(w, P)
$$

to (4)-(9), where $s_{\min }$ is a scalar representing the minimum value desired for the severity of the system disturbance. Thus we seek to obtain only those solutions which result in system partitions having power imbalances greater than $\sqrt{s_{\min }}$ in magnitude.

The constrained optimization formulation presented in Section III-B, along with (13), seeks to minimize the (effective) number of line removals while ensuring the disturbance severity is greater than some threshold. Alternatively, the problem may be reformulated to maximize 
the severity while ensuring that the (effective) number of line removals is less than some threshold.

We note that (13) is not necessarily an accurate measure of disturbance severity when a solution partitions the graph into three or more groups. We are currently investigating ways to extend this work. In this paper we focus on disturbances that essentially break the system into two parts. The results presented in Section IV correspond to cases when there are exactly two partitions.

\section{Test Problem - The IEEE 30 Bus System}

The ideas outlined in Sections I-III are tested using the IEEE 30 bus system. This system has 6 generators and 41 lines [7]. To solve the constrained optimization problems described in Section III, that is, with the objective of either minimizing the (effective) number of line removals, or maximizing the severity of the failure, we are currently using the solvers available from the NEOS server [8,9], in particular, KNITRO [10, 11], IPOPT [12, 13] and LOQO [14]. These are interior-point methods for the solution of constrained optimization problems with nonlinear objective functions and/or constraints.

A number of solutions have been found for which removal of a small number of lines $(\leq 6)$ partitions the network into two groups, resulting in a severe failure in the sense defined in Section III-C. Figure 3 graphically presents one such solution, when a removal of four lines results in a power shortage/surplus of 8.43 p.u. in each group (island). Note that this power mismatch corresponds to a severity of 71.07 (Section III-C).

Given the solution vectors to the optimization problem (Sections III-B and C), namely $\gamma, \theta$ and $w$, one is able to readily identify from the latter the decomposition of the buses into the disjoint groups: buses 1 through 21, and 28 belong to one group, with the remaining buses lying in the other group. Recall also (Section III-B) that, in our formulation, lines in the partition cut-set are those for which either an element in $\gamma$ is equal to 1 , or the phase angle across them is $\pm \pi / 2$ (maximum power transfer). For the solution depicted in Figure 3, all lines in the cut-set, namely those between buses 10-22, 21-22, 1523 , and 27-28, have the phase angles across them equal to $-\pi / 2$. The corresponding elements in $\gamma$ have the values 0.72 , $0.97,0.54$, and 0 , respectively. There are two other lines identified by the solution as important, namely, those between buses $6-28$ and $8-28$, as their $\gamma_{i}$ are 1 and 0.16 respectively. The phase angles across them are much less than $\pi / 2$ in magnitude. These lines, however, do not lie on the partition cut-set.

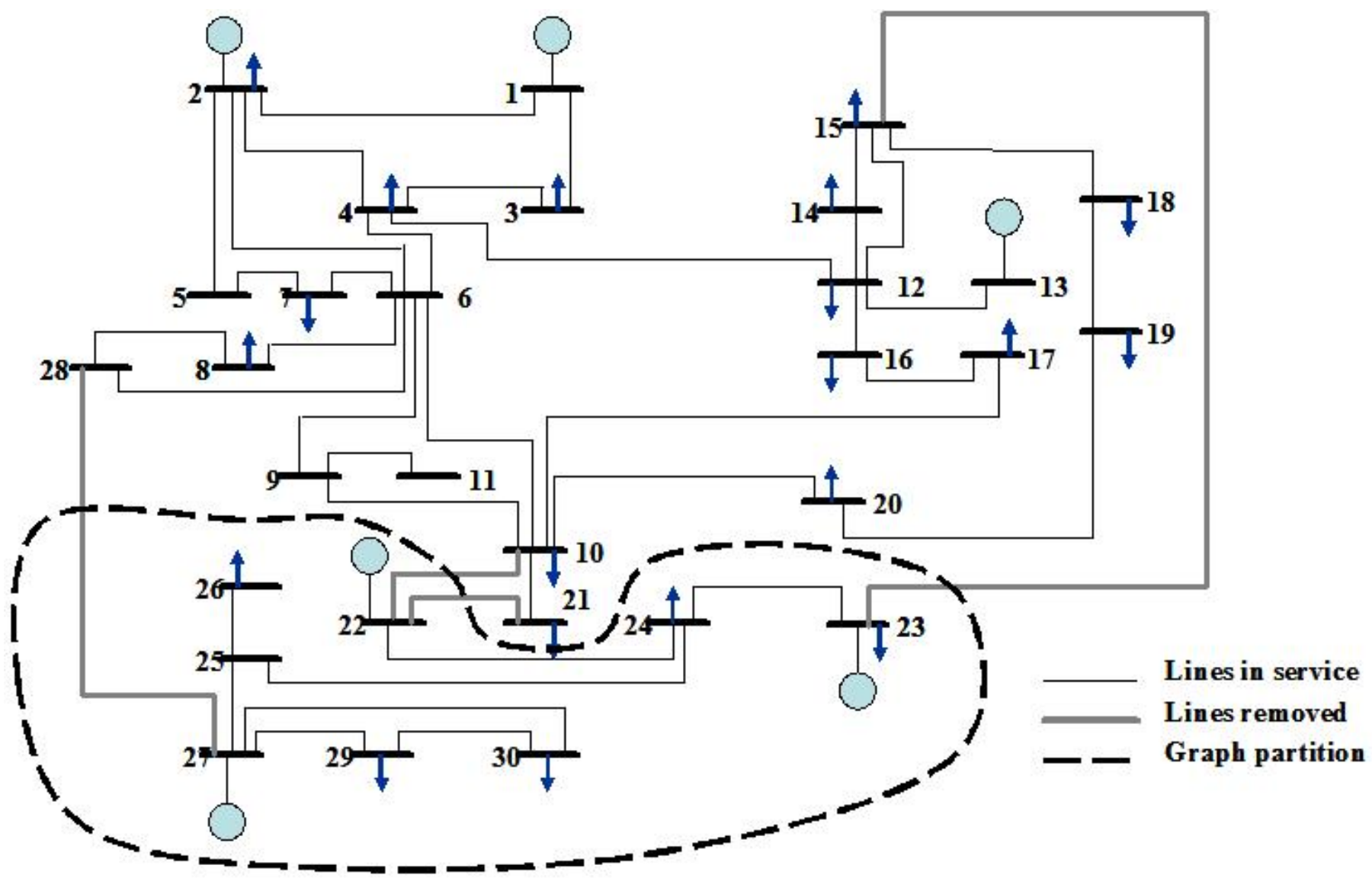

Figure 3: The IEEE 30 bus system partitioned into two groups, with a power shortage/surplus of 8.43 p.u. in each group. 
We emphasize that this exercise of solving the relaxed optimization problem provides us with a set of lines, much fewer in number than the total number of lines in the network, for further detailed analysis. For the case under consideration, this set comprises the lines between buses 10-22, 21-22, 15$23,27-28,6-28$ and 8-28. A detailed analysis may include either testing whether or not a feasible power flow solution exists when different subsets of lines from the aforementioned set are removed from the network (done by enumeration), or the use of more sophisticated tools, such as combinatorial algorithms. For instance, power flow solution existence test by enumeration provides the following observations. Power flow solution does not exist (at least locally) when the line between buses 27-28 is removed by itself. Moreover, removal of pairs of lines at a time, namely, between buses 10-22 and 21-22, between 21-22 and 15-23, and between 6-28 and 8-28, while keeping other lines intact, leads to power flow equation insolvability. Thus, the power system is most vulnerable to the operating statuses of these lines. Note that although the power flow solution (locally) does not exist, the graph remains connected. A measure of severity will be needed to isolate cases, out of the ones just described that produce large disturbances without partitioning the graph. The ensuing disturbances after the loss of the identified lines may be more or less severe than indicated by our severity function. A detailed dynamic simulation including control and protection devices would be necessary for a detailed assessment. As previously noted, the simple measure described in Section III$\mathrm{C}$ is intended for screening purposes and is inadequate for detailed analysis.

Apart from the solution described by Figure 3, other solutions that partition the network into two groups have been found. Specific features of these solutions are summarized in Table 1, where the list is arranged in increasing order by the value of the severity function. Note that the size of the partition cut-sets ranges between 2 and 6 lines, so in each case the number of lines identified by a solution is much smaller than the total number of lines, i.e., 41 , in the network. The severity of the resulting system failure is reasonably large in each case, although there is no apparent correlation between the size of the cut-sets and the corresponding severity.

TABLE I

PARTITIONING OF THE IEEE 30 Bus System INTO Two Groups

\begin{tabular}{ccccc}
\hline \hline $\begin{array}{c}\text { Solution } \\
\text { Id }\end{array}$ & severity $(w, P)$ & $\begin{array}{c}\text { Size of cut-set } \\
\text { (\# of lines) }\end{array}$ & $\operatorname{trace}(\Gamma)$ & $\begin{array}{c}\text { \# of buses } \\
\text { in groups }\end{array}$ \\
\hline 1 & 5.15 & 6 & 5.0920 & 12,18 \\
2 & 9.25 & 2 & 2.0383 & 5,25 \\
3 & 12.82 & 4 & 3.6787 & 8,22 \\
4 & 14.06 & 6 & 5.3235 & 12,18 \\
5 & 24.01 & 4 & 3.9106 & 11,19 \\
6 & 25.20 & 6 & 4.9997 & 9,21 \\
7 & 25.94 & 6 & 5.1301 & 8,22 \\
8 & 28.41 & 4 & 3.7554 & 7,23 \\
9 & 71.07 & 4 & 3.3811 & 8,22 \\
\hline \hline
\end{tabular}

\section{DiSCUSSION AND CONCLUSIONS}

In this paper we have proposed a well-defined optimization problem to help screen for severe multiple contingencies. The approach appears effective in the small system we have studied and its properties should scale well.

The computational burden involved in a complete enumeration of all possible combinations is greatly reduced using the screening process. Our example contains 41 lines. A combinatorial analysis of all combinations of three simultaneous line outages would require 10660 separate calculations. After the screening process identifies, say, six lines, the combinations reduce to 20 .

Our screening process is essentially a graph partitioning algorithm. One can generally expect that a largely planar graph with $n$ branches will separate with a cut-set of size $\sqrt{n}$. Given a model of 10000 lines, a screening process that yields 100 lines of interest will enable combinatorial analysis using very fast computers. (A combinatorial analysis of three events reduces from $1.6 \times 101^{11}$ to 161,700 . A supercomputer with 10,000 processors could handle the latter easily, but the former remains impossible.)

There are some impediments to this approach that require further research. The optimization problem is nonconvex. The results of this are clearly seen in the results presented in Table I. We are presently seeking ways to promote convergence to solutions with reasonably high severities. Nevertheless, the solutions that are found that satisfy the stated severity level are interesting in their own right, even though they may not be the most severe.

The model used in the screening process is crude. It neglects voltage variations and reactive power considerations. We intend to extend the approach to consider these issues. If we ignore the severity function, the extension in the optimization framework is straightforward; however, the structure of the problem loses the direct relations to spectral graph theory. The solutions found will be on the edge of a region of feasibility. Incorporating a severity function into the optimization problem is important so as to avoid trivial or solutions of little consequence. A new severity function will need to be developed to replace the one in this paper, which has roots in spectral graph theory.

Since our screening process is a graph partitioning algorithm, it is essential to consider other alternatives to the optimization approach followed here. We are doing so, but the reader should recognize that this problem falls outside the realm of traditional graph partitioning algorithms. It is a dual objective problem. We wish to minimize the number of cuts while simultaneously ensuring that the power disrupted by such a cut exceeds a predefined value. Despite the language used in this description, this problem should not be confused with the well-known min-cut, max flow problem in which the maximum flow between two points can be determined by finding the minimum weighted cut that separates the points. Our problem is very different (we do not know the points $a$ priori, for instance) and it is more difficult. We have not yet 
identified or developed any graph partitioning algorithms that work better for this problem than the approach in this paper, but we continue to pursue this avenue of research.

\section{APPENDIX}

This appendix lists the IEEE 30 bus system parameters used for the study presented in this paper. The system is assumed to be lossless and the reactive power flows are ignored.

TABLE II

Bus DATA FOR THE IEEE 30 BUS SYSTEM

\begin{tabular}{|c|c|c|}
\hline Bus number & $\begin{array}{l}\text { Active power } \\
\text { generation }(\mathrm{MW})\end{array}$ & $\begin{array}{l}\text { Active power } \\
\text { load (MW) }\end{array}$ \\
\hline 1 & 135.4 & 000.0 \\
\hline 2 & 209.7 & 217.0 \\
\hline 3 & 000.0 & 024.0 \\
\hline 4 & 000.0 & 076.0 \\
\hline 5 & 000.0 & 000.0 \\
\hline 6 & 000.0 & 000.0 \\
\hline 7 & 000.0 & 228.0 \\
\hline 8 & 000.0 & 300.0 \\
\hline 9 & 000.0 & 000.0 \\
\hline 10 & 000.0 & 058.0 \\
\hline 11 & 000.0 & 000.0 \\
\hline 12 & 000.0 & 112.0 \\
\hline 13 & 410.0 & 000.0 \\
\hline 14 & 000.0 & 062.0 \\
\hline 15 & 000.0 & 082.0 \\
\hline 16 & 000.0 & 035.0 \\
\hline 17 & 000.0 & 090.0 \\
\hline 18 & 000.0 & 032.0 \\
\hline 19 & 000.0 & 095.0 \\
\hline 20 & 000.0 & 022.0 \\
\hline 21 & 000.0 & 175.0 \\
\hline 22 & 315.9 & 000.0 \\
\hline 23 & 342.0 & 032.0 \\
\hline 24 & 000.0 & 087.0 \\
\hline 25 & 000.0 & 000.0 \\
\hline 26 & 000.0 & 035.0 \\
\hline 27 & 469.1 & 000.0 \\
\hline 28 & 000.0 & 000.0 \\
\hline 29 & 000.0 & 024.0 \\
\hline 30 & 000.0 & 106.0 \\
\hline
\end{tabular}

TABLE III LINE DATA FOR THE IEEE 30 BUS SYSTEM

From Bus number To Bus number Line Reactance

\begin{tabular}{lll} 
& & (p.u.) \\
\hline 1 & 2 & 0.06 \\
1 & 3 & 0.19 \\
2 & 4 & 0.17 \\
3 & 4 & 0.04
\end{tabular}

\begin{tabular}{|c|c|c|}
\hline 2 & 5 & 0.20 \\
\hline 2 & 6 & 0.18 \\
\hline 4 & 6 & 0.04 \\
\hline 5 & 7 & 0.12 \\
\hline 6 & 7 & 0.08 \\
\hline 6 & 8 & 0.04 \\
\hline 6 & 9 & 0.21 \\
\hline 6 & 10 & 0.56 \\
\hline 9 & 11 & 0.21 \\
\hline 9 & 10 & 0.11 \\
\hline 4 & 12 & 0.26 \\
\hline 12 & 13 & 0.14 \\
\hline 12 & 14 & 0.26 \\
\hline 12 & 15 & 0.13 \\
\hline 12 & 16 & 0.20 \\
\hline 14 & 15 & 0.20 \\
\hline 16 & 17 & 0.19 \\
\hline 15 & 18 & 0.22 \\
\hline 18 & 19 & 0.13 \\
\hline 19 & 20 & 0.07 \\
\hline 10 & 20 & 0.21 \\
\hline 10 & 17 & 0.08 \\
\hline 10 & 21 & 0.07 \\
\hline 10 & 22 & 0.15 \\
\hline 21 & 22 & 0.02 \\
\hline 15 & 23 & 0.20 \\
\hline 22 & 24 & 0.18 \\
\hline 23 & 24 & 0.27 \\
\hline 24 & 25 & 0.33 \\
\hline 25 & 26 & 0.38 \\
\hline 25 & 27 & 0.21 \\
\hline 27 & 28 & 0.40 \\
\hline 27 & 29 & 0.42 \\
\hline 27 & 30 & 0.60 \\
\hline 29 & 30 & 0.45 \\
\hline 8 & 28 & 0.20 \\
\hline 6 & 28 & 0.06 \\
\hline
\end{tabular}

\section{ACKNOWLEDGMENT}

We would like to thank Prof. Gerald Heydt at Arizona State University, Phoenix, for his helpful comments on this work.

\section{REFERENCES}

[1] U.S.-Canada Power System Outage Task Force, "Final report on the August 14, 2003 blackout in the United States and Canada: causes and recommendations," April 2004.

[2] Electric Consumer Research Council (ELCON), "The Economic Impacts of the August 2003 Blackout," February 2004.

[3] F. Alvarado, I. Dobson, and Y. Hu, "Computation of closest bifurcations in power systems," IEEE Transactions on Power Systems, Vol. 9, No. 2, pp. 918-928, May 1994. 
[4] F. R. K. Chung, Spectral Graph Theory, Providence, RI: Amer. Math. Soc., 1997.

[5] J. Demmel, “CS 267: Notes for Lecture 23, April 9, 1999. Graph

Partitioning, Part 2. Available:

http://www.cs.berkeley.edu/ demmel/cs267/lecture20/lecture20.html.

[6] System disturbance reports, North American Electric Reliability Council, Disturbance Analysis Working Group. Available: http://www.nerc.com/ dawg/.

[7] Power Systems Test Case Archive. Available: http://www.ee.washington.edu/research/pstca/.

[8] J. Czyzyk, M. Mesnier, and J. Moré, "The NEOS server," IEEE Journal on Computational Science and Engineering, vol. 5, pp. 68-75, 1998.

[9] NEOS server for optimization. Available: http://www-eos.mcs.anl.gov/.

[10] R. H. Byrd, M. E. Hribar, and J. Nocedal, "An interior point algorithm for large scale nonlinear programming," SIAM Journal on Optimization, vol. 9, no. 4, pp. 877-900, Sept. 1999.

[11] KNITRO. Available: http://www.ziena.com/knitro.html.

[12] A. Wächter and L. T. Biegler, "On the implementation of a primal-dual interior point filter line search algorithm for large-scale nonlinear programming," Research Report, IBM T. J. Watson Research Center, Yorktown, USA, (March 2004 - accepted for publication in Mathematical Programming).

[13] IPOPT: a software package for large-scale nonlinear optimization. Available: http://www.coin-or.org/Ipopt/.

[14] LOQO. Available:

http://www-neos.mcs.anl.gov/neos/solvers/NCO:LOQO-AMPL/. 